\title{
QUANTUM LOGIC AND THE LOCALLY CONVEX SPACES
}

\author{
BY
}

\author{
W. JOHN WILBUR
}

\begin{abstract}
An important theorem of Kakutani and Mackey characterizes an infinite dimensional real (complex) Hilbert space as an infinite dimensional real (complex) Banach space whose lattice of closed subspaces admits an orthocomplementation. This result, also valid for quaternionic spaces, has proved useful as a justification for the unique role of Hilbert space in quantum theory. With a like application in mind, we present in the present paper a number of characterizations of real and complex Hilbert space in the class of locally convex spaces. One of these is an extension of the Kakutani-Mackey result from the infinite dimensional Banach spaces to the class of all infinite dimensional complete Mackey spaces. The implications for the foundations of quantum theory are discussed.
\end{abstract}

1. Introduction. Since the fundamental work of Birkhoff and von Neumann [3] on the logic of quantum mechanics, it has been recognized that a correct model for quantum logic must incorporate essential features of the finite dimensional projective geometries. In a general way the lattice of closed subspaces of a Hilbert space with its natural orthocomplementation is indicated. However, there has remained the problem of adequate justification for excluding logics which might be obtained in a similar manner from other topological vector spaces and for excluding other topological vector spaces which might yield the same logic as a Hilbert space. Important progress on this problem is represented by results of Kakutani and Mackey [6], [7] which characterize an infinite dimensional real or complex Hilbert space as an infinite dimensional real or complex Banach space whose lattice of closed subspaces admits an orthocomplementation. It is our purpose in the present paper to pursue the problem further, as suggested by Varadarajan $[11, \mathrm{p} .143]$, and to investigate the unique position of infinite dimensional Hilbert space in the class of all real and complex topological vector spaces.

Our results may be briefly summarized as follows. We begin in $\S 3$ by showing

Received by the editors August 16, 1973.

AMS (MOS) subject classifications (1970). Primary 46A05, 46C05, 50D20, 50D25, 81A12; Secondary 06A25, 54H10.

Key words and phrases. Quantum logic, projective logic, state, question lattice, lattice of closed subspaces, orthocomplementation, $\theta$-bilinear symmetric form, continuous automorphism, discontinuous automorphism, $\theta$-bilinear space, Hilbertian space, inner product space, Hilbert space, metrizable space, Mackey space. 
that if there is an orthocomplementation on the lattice of closed subspaces of a topological vector space, either real or complex, then there is a locally convex topology on the underlying vector space which produces the same lattice of closed subspaces. Though this result is rather elementary, it serves to reduce the problem to the consideration of locally convex spaces. The contents of $\S \S 4$ and 5 are technical results concerning logic and orthocomplementation structures on locally convex spaces, which are employed in $\S 6$. The main theorem of $\S 4$ states that a logic based on the lattice of closed subspaces of a locally convex space is a projective logic. Topological conditions are given in $\$ 5$ which insure that all field automorphisms corresponding to orthocomplementations on a given complex locally convex space shall be continuous. In $\S 6$ the results of $\S \S 4$ and 5 are applied to obtain several characterizations of an infinite dimensional real or complex Hilbert space. An infinite dimensional Hilbert space is characterized as:

(1) an infinite dimensional complete Mackey space which admits an orthocomplementation on its lattice of closed subspaces;

(2) an infinite dimensional Mackey space whose topology is properly stronger than its weak topology and whose lattice of closed subspaces admits an orthocomplementation making it a logic;

(3) an infinite dimensional metrizable space whose lattice of closed subspaces admits an orthocomplementation making it a logic.

One consequence of these characterizations is that the only infinite dimensional locally convex spaces other than Hilbert spaces which allow a logic structure on their lattice of closed subspaces are spaces with very weak and exotic topologies. (Some examples are given in \$7.) This, together with pertinent topological aspects of physical theory, leads in $\S 8$ to the conclusion that the Hilbert spaces are the only locally convex spaces which meet the minimal technical necessities of quantum theory.

2. Preliminaries. Fundamental to the question lattice approach to quantum theory is the concept of an orthocomplementation of a complemented lattice. A lattice $L$ with 0 and 1 is said to be complemented if for any $a \in L$ there is a $b \in L$ satisfying

(i) $a \wedge b=0$,

(ii) $a \vee b=1$.

The element $b$, which may not be unique, is called a complement of $a$. If $L$ is a complemented lattice, a self-mapping ' which takes each element of $L$ to one of its complements and also satisfies

(i) $a<b$ implies $b^{\prime}<a^{\prime}$,

(ii) $a^{\prime \prime}=a$,

for any $a$ and $b$ in $L$, is called an orthocomplementation. There are some useful 
refinements of these notions. A lattice $L$ with an orthocomplementation ' is said to be a logic if every countable subset of $L$ has a supremum and an infimum and if for $a, b \in L$ with $a<b$ there is always a $c \in L$ with $c<a^{\prime}$ and $a \vee c=b$. It is generally assumed that the question lattice of quantum theory has the structure of a logic. For the somewhat technical definition of a projective logic, which we shall not use directly, we refer the reader to Varadarajan [11].

We shall be dealing largely with lattices of closed subspaces of locally convex spaces. If $E$ is any topological vector space, real or complex, $C(E)$ will denote its lattice of closed subspaces. Of particular importance from the lattice theoretic viewpoint are those spaces whose topologies are Hilbert space topologies, i.e., those spaces which are topologically isomorphic to a Hilbert space treated as a topological vector space. We shall say that such a space may be realized as a Hilbert space. These are special conventions. For the standard concepts and constructs of the theory of topological vector spaces we refer to Schaefer [9]. As a matter of convenience, it will be assumed in the sequel that all topological vector spaces mentioned are either real or complex and of dimension at least four. Throughout, $K$ will be used to denote either the real or the complex field.

Let $D$ be a division ring and $V$ a vector space over $D$ of dimension at least four. Let $\theta$ be an involutive antiautomorphism of $D$. A form $\langle$, $\rangle$ on $V \times V$ is called $\theta$-bilinear symmetric provided

(i) $\langle a x+b y, z\rangle=a\langle x, z\rangle+b\langle y, z\rangle$,

(ii) $\langle z, a x+b y\rangle=\langle z, x\rangle \theta(a)+\langle z, y\rangle \theta(b)$,

(iii) $\langle x, y\rangle=\theta(\langle y, x\rangle)$,

for any $x, y, z \in V$ and $a, b \in D$. If $\langle$,$\rangle is also definite in that \langle x, x\rangle=0$ if and only if $x=0$ we shall call the pair $(V,\langle\rangle$,$) a \theta$-bilinear space. The term inner product space will be reserved for the case when $D$ is either the real or complex field and correspondingly $\theta$ is either the identity or the complex conjugation. The basic structure that we have here called a $\theta$-bilinear space was originally discovered by Birkhoff and von Neumann [3] as characterizing an orthocomplementation on the lattice of subspaces of a finite dimensional vector space. It has also proved fruitful in infinite dimensional spaces and will play a central role in our discussion. A $\theta$-bilinear space is suggestive of Hilbert space, and accordingly we shall say $\boldsymbol{x}$ and $y$ are orthogonal (also written $x \perp y$ ) if $\langle x, y\rangle=0$ and $x$ is normalized if $\langle x, x\rangle=1$. The annihilator of a subspace $M$ of $V$ will be the subspace

$$
M^{\perp}=\{x \in V \mid\langle x, y\rangle=0 \text { for all } y \in M\} .
$$

A subspace $M$ is called $\langle$,$\rangle -closed [11] if and only if M=M^{\perp 1}$. The collection of all $\langle$,$\rangle -closed subspaces of V$ forms a complete lattice $L(V,\langle\rangle$,$) which con-$ tains all finite dimensional subspaces of $V$, and $\perp$ is an orthocomplementation on 
$L(V,\langle\rangle$,$) . Finally (V,\langle\rangle$,$) is said to be Hilbertian if for any M \in L(V,\langle\rangle$,$) ,$ $V=M+M^{\perp}$ algebraically. For the details, see [11, Chapter VII, §5].

3. Reduction to locally convex spaces. Since we shall be concerned mainly with locally convex topological vector spaces, we shall here answer the question whether something might not be gained by considering the more general class of topological vector spaces over the real or complex field. The answer is that one obtains no new lattices with orthocomplementations and hence no new potential models for quantum mechanics. After this section, we shall restrict our attention completely to the locally convex spaces.

Let $E$ be a topological vector space and let ' be an orthocomplementation on $C(E)$. Then in accordance with a lemma of Varadarajan [11,7.2], there are a $\theta$ and a $\theta$-bilinear symmetric form $\langle$,$\rangle on E \times E$ making $(E,\langle\rangle$,$) a \theta$-bilinear space with the property that for any $x \in E,(K \circ x)^{\prime}=(K \circ x)^{\perp}$.

3.1. Lemma. $C(E)=L(E,\langle\rangle$,$) and { }^{\prime}=1$.

Proof. Let $M \in L(E,\langle\rangle$,$) . Then$

$$
M=\left(M^{\perp}\right)^{\perp}=\bigcap(K \circ x)^{\perp}\left(x \in M^{\perp}\right)=\bigcap(K \circ x)^{\prime}\left(x \in M^{\perp}\right) .
$$

Thus $M \in C(E)$ because $(K \circ x)^{\prime} \in C(E)$ for each $x \in M^{\perp}$. Now suppose $M \in C(E)$. Then

$$
\begin{aligned}
M^{\prime} & =(\bigvee\{(K \circ x) \mid x \in M\})^{\prime}=\bigwedge\left\{(K \circ x)^{\prime} \mid x \in M\right\} \\
& =\bigwedge\left\{(K \circ x)^{\perp} \mid x \in M\right\}=\bigcap\left\{(K \circ x)^{\perp} \mid x \in M\right\}=M^{\perp} .
\end{aligned}
$$

Repeating this gives $M=M^{\prime \prime}=M^{\perp \perp}$ and $M \in L(E,\langle\rangle$,$) .$

This simple result shows that $\langle$,$\rangle is very strongly involved with the topology$ on $E$ and leads us toward the desired conclusion.

Now for each $x \in E,\langle\circ, x\rangle$ defines a linear functional on $E$ which is denoted by $\beta_{x}$ (see [11, p. 137]). Since the kernel of $\beta_{x}$ is $(K \circ x)^{\perp}=(K \circ x)^{\prime}$ and thus closed, each $\beta_{x}$ is continuous and therefore in the topological dual $F$ of $E$.

3.2. Lemma. The mapping $\beta: x \rightarrow \beta_{x}$ is a $\theta$-linear bijection from $E$ onto $F$.

The proof is essentially that of a similar lemma for Banach spaces given in $[11,7.3]$.

3.3. THEOREM. If there is an orthocomplementation on $\mathrm{C}(E)$ then there is a locally convex topology $\tau$ for $E$ with $C(E)=C\left(E_{\tau}\right)$ (here $E_{\tau}$ is $E$ with the topology $\tau$ ).

Proof. Let $(E,\langle\rangle$,$) be the \theta$-bilinear space associated with the orthocomplementation on $C(E)$. Because $\beta_{x}(x)=\langle x, x\rangle \neq 0$ if $x \neq 0$, it is clear by 3.2 
that $E$ and its topological dual $F$ comprise a duality in the sense of [9, Chapter IV]. Let $E_{\tau}$ be $E$ with the Mackey topology induced by $F$ (any locally convex topology consistent with the duality would serve as well). If $M$ is in $C(E)$ then, by 3.1, $M=M^{\perp \perp}$ and $M$ is the intersection of the kernels of elements in $\beta\left(M^{\perp}\right)$ and hence is in $C\left(E_{\tau}\right)$. Conversely, if $M$ is in $C\left(E_{\tau}\right)$ then by the Hahn-Banach theorem it is the intersection of the kernels of some subset of $F$, and so by 3.2 and 3.1 must be in $C(E)$. Thus $C\left(E_{\tau}\right)=C(E)$.

From now on we shall assume that $E$ is a locally convex space and that $F$ is its topological dual.

4. Orthocomplementations and logics. By 3.1 if ' is an orthocomplementation on $C(E)$ then there is a $\theta$-bilinear space $(E,\langle\rangle$,$) with C(E)=L(E,\langle, \downarrow)$ and $'=1$. Conversely if $V$ is a vector space over $K$ and $(V,\langle\rangle$,$) is a \theta$-bilinear space, one may let $F$ be the space of functionals $\langle\circ, x\rangle, x \in V$, and let $E$ be $V$ with the Mackey topology induced by $F$. Then $C(E)=L(V,\langle\rangle$,$) and \perp$ is an orthocomplementation on $C(E)$. This construction is inherent in the proof of 3.3. Since the lattice $C(E)$ with its orthocomplementation uniquely determines the $\theta$-bilinear space $(V,\langle\rangle$,$) except for an indefiniteness in \langle$,$\rangle by a constant multiple [11],$ there follows the essential equivalence of orthocomplementations on lattices $C(E)$ and of $\theta$-bilinear spaces over the real and complex fields, of a topological-lattice theoretic object and an algebraic object. It is our purpose in this section to extend this equivalence in a simple form to logics constructed on lattices $C(E)$.

4.1. THEOREM. Let $V$ be a vector space over $K$ and let $(V, \zeta\rangle$,$) be a \theta$-bilinear space. Then if $L(V,\langle\rangle$,$) with the orthocomplementation \perp$ is a logic, $(V,\langle\rangle$,$) is Hilbertian.$

Proof. Suppose $L(V,\langle\rangle$,$) is a logic and let M$ be in $L(V,\langle\rangle$,$) . We must$ show $V=M+M^{\perp}$ algebraically. Let $x \in V$ and let us assume $x \notin M$. Let $E$ be $V$ with the Mackey topology induced by $\langle$,$\rangle . Then C(E)=L(V,\langle\rangle$,$) and$ $M \in C(E)$ and $K \circ x \in C(E)$. Now in any topological vector space over a topologically complete field the algebraic sum of a closed subspace and a finite dimensional subspace is closed. Thus $M+K \circ x \in C(E)=L(V,\langle\rangle$,$) . Since L(V,\langle\rangle$,$) is a$ logic under $\perp$, there is an $N \in L(V,\langle\rangle$,$) with M \perp N$ and $M \vee N=M \vee(K \circ x)=$ $M+K \circ x$. Here $M$ is a maximal proper subspace of $M+K \circ x$ so we must have $N=K \circ y$ for some $y$. Again employing the argument on closed subspaces, $M \vee N=M+N$. Thus $M+N=M+K \circ x$. It follows that $x=m+n$ for some $m \in M$ and $n \in N$ and $x \in M+M^{\perp}$. As a result, $V=M+M^{\perp}$ and $(V,\langle\rangle$, is Hilbertian.

The topology plays an important part in this proof, and hence we are limited to the real or complex fields. Whether the result actually holds over an arbitrary 
division ring, we do not know. In any case, the converse that if $(V,\langle\rangle$,$) is Hil-$ bertian then $L(V,\langle\rangle$,$) is a logic is known for arbitrary division rings [11, 7.40].$ There follows the desired simple equivalence, namely, an orthocomplementation on $C(E)$ making it a logic is equivalent to a $\theta$-bilinear symmetric form $\langle$,$\rangle on E$ for which $C(E)=L(E,\langle\rangle$,$) and (E,\langle\rangle$,$) is Hilbertian. A further consequence is$ the fact that the class of logics which can be constructed on the lattices $C(E)$ is just the class of complete projective logics over the real or complex fields [11, p. 176 and 7.40].

We shall close this section with a result which shows how the fact that $L(V,\langle\rangle$,$) is a logic influences the topology induced on the corresponding locally$ convex space $E$. First some general considerations will be needed concerning the dual $F$ of $E$. If $\beta_{x}$ and $\beta_{y}$ are in $F$, define $\left(\beta_{x}, \beta_{y}\right)=\langle y, x\rangle$. Then $($,$) is a \theta$ bilinear symmetric form on $F$. Further, it is easy to see that $\beta$ and $\beta^{-1}$ preserve annihilators. Thus $\beta$ induces an isomorphism between $L(E,<\rangle$,$) and L(F,()$,$) ,$ which preserves complementations, and if $L(E,\langle\rangle$,$) is a logic so is L(F,()$,$) .$ Now by the bipolar theorem, the closed subspaces of $F$ are characterized as those subspaces which equal their own bipolars, and since for any subspace $M$ of $F$, $M^{\infty}=M^{\perp \perp}$, the closed subspaces of $F$ are just the (, )-closed subspaces. It follows that the Mackey topology induced on $F$ by $E$ is the same as the Mackey topology induced on $F$ by $(F,()$,$) . This places E$ and $F$ on an equal basis.

4.2. Proposition. Let $E$ be a Mackey space with an orthocomplementation on $C(E)$ and let $(E,\langle\rangle$,$) be the corresponding \theta$-bilinear space. If $M \in C(E)$ and $E=M+M^{\perp}$ algebraically then the sum is also topological.

Proof. First we shall show that $E=M+M^{\perp}$ is topological for the weak topology. According to $[9$, p. 20, 2.2], it is sufficient to show that the projection onto $M$ associated with $M+M^{\perp}$ is weakly continuous. Let $u$ represent this projection. The weak continuity of $u$ will follow when we show that the dual map $u^{*}$ maps $F$ into $F$. Let $\beta_{y} \in F$. Then $y=b+c$ with $b \in M$ and $c \in M^{\perp}$ and $u^{*}\left(\beta_{y}\right)=\beta_{y} \circ u=\beta_{b}$. Thus $u$ is weakly continuous and $M+M^{\perp}$ is topological in the weak topology on $E$.

Now $F=\beta(E)=\beta(M)+\beta(M)^{\perp}$ and by a repetition of the argument just given, the projection $u^{*}$ from $F$ onto $\beta(M)$ is weakly continuous in $F$. It follows that $u^{*}$ carries weakly compact, convex, circled subsets of $F$ into weakly compact, convex, circled subsets of $F$ and by [9, p. 130, 2.4], $u$ must be continuous in the Mackey topology. Again by $[9$, p. $20,2.2]$, we have $E=M+M^{\perp}$ topologically, this time in the Mackey topology.

We see from this result that if $E$ is a Mackey space and there is an orthocomplementation on $C(E)$ making it a logic then there must be a continuous projection 
onto each closed subspace of $E$. Though we shall not pursue the matter further, this is one of the important properties of Hilbert space.

5. Continuity of the automorphism. Let $E$ be a locally convex space with an orthocomplementation on $C(E)$ and let $(E,\langle\rangle$,$) be the corresponding \theta$-bilinear space. If $K=\mathbf{R}$ then $\theta$ must be the identity and $(E, \zeta\rangle$,$) is an inner product$ space and $\langle$,$\rangle may be used to convert the space E$ into a real pre-Hilbert space. If $K=C$ the situation is much more difficult. However, it is known [7] that if $\theta$ is continuous then it is just conjugation and $\langle$,$\rangle allows E$ to assume a complex pre-Hilbert structure. In obtaining their classic characterizations of complex Hilbert space, Kakutani and Mackey [7] use a device of Arnold [1] to show that when $E$ is infinite dimensional and normable $\theta$ must be continuous. It is our purpose in this section to show that the same is true for large classes of infinite dimensional locally convex spaces.

\subsection{THEOREM. If the Mackey topology is stronger than the weak topology} in either $E$ or $F$, then $\theta$ must be continuous.

Proof. We shall give the proof assuming that the Mackey topology of $E$ is stronger than the weak topology. The same argument would apply to $F$ because $\theta$ is involved equally in the $\theta$-bilinear spaces $(E,\langle\rangle$,$) and (F,()$,$) . If the Mackey$ topology on $E$ is stronger than the weak topology then by $[9$, p. 131] there is an infinite dimensional weakly compact, convex, circled subset $Q$ of $F$. Let $\left\{\alpha_{n}\right\}_{n=1}^{\infty}$ be a linearly independent sequence from $Q$. Then since $\langle$,$\rangle is definite in the sense$ that $\langle\alpha, \alpha\rangle=0$ if and only if $\alpha=0$, we can apply the Gram-Schmidt technique to $\left\{\alpha_{n}\right\}_{n=1}^{\infty}$ to obtain another sequence $\left\{\gamma_{n}\right\}_{n=1}^{\infty}$ of nonzero elements such that $\left\langle\gamma_{n}, \gamma_{m}\right\rangle=0$ if $n \neq m$ and each $\gamma_{n}$ is a finite linear combination of the $\alpha$ 's. Now $Q$ is both convex and circled. Thus for some sequence $\left\{s_{n}\right\}_{n=1}^{\infty}$ of nonzero numbers, $\left\{s_{n} \gamma_{n}\right\}_{n=1}^{\infty}$ is a sequence from $Q$. If we let $(1 / n) s_{n} \gamma_{n}=\beta_{x_{n}}$ then $\left\{\beta_{x_{n}}\right\}_{n=1}^{\infty}$ is a sequence from $Q$ which converges weakly to zero and satisfies $\left(\beta_{x_{n}}, \beta_{x_{m}}\right)=0$ if and only if $n \neq m$. Now suppose $\theta$ is discontinuous. Then there must be a sequence $\left\{c_{n}\right\}_{n=1}^{\infty}$ which converges to zero with zero not even a cluster point of $\left\{\theta\left(c_{n}\right)\right\}_{n=1}^{\infty}$. We define recursively a subsequence $\left\{c_{n(i)}\right\}_{i=1}^{\infty}$ of $\left\{c_{n}\right\}_{n=1}^{\infty}$ as follows. Let $c_{n(1)}$ be the first $c_{n}$ with $\left|c_{n} /\left\langle x_{1}, x_{1}\right\rangle\right|<2^{-1}$. If $c_{n(k-1)}$ has been defined let $c_{n(k)}$ be the first $c_{n}$ with $\left.n\right\rangle n(k-1)$ and $\left|c_{n}\right|\left\langle x_{k}, x_{k}\right\rangle \mid<2^{-k}$. Also define the sequence $\left\{\lambda_{i}\right\}_{i=1}^{\infty}$ by $\lambda_{i}=c_{n(i)} /\left\langle x_{i}, x_{i}\right\rangle$ for all $i$. Because $\Sigma\left|\lambda_{i}\right|<1$ and $Q$ is convex, circled, and weakly compact, $\Sigma \lambda_{i} \beta_{x_{i}}$ is weakly convergent in $Q$ to some $\beta_{u}$. Thus for each $j$,

$$
\left\langle x_{j}, u\right\rangle=\beta_{u}\left(x_{j}\right)=\lim _{n \rightarrow \infty} \sum_{i=1}^{n} \lambda_{i} \beta_{x_{i}}\left(x_{j}\right)=\lambda_{j}\left\langle x_{j}, x_{j}\right\rangle=c_{n(j)} .
$$


By the symmetry of $\langle$,

$$
\beta_{x_{j}}(u)=\left\langle u, x_{j}\right\rangle=\theta\left(\left\langle x_{j}, u\right\rangle\right)=\theta\left(c_{n(j)}\right) .
$$

But $\beta_{x_{j}}$ converges weakly to zero while $\theta\left(c_{n(j)}\right)$ does not converge to zero since $\left\{\theta\left(c_{n}\right)\right\}_{n=1}^{\infty}$ did not have zero as a cluster point. This contradiction establishes the theorem.

Since the map $\beta: E \rightarrow F$ is $\theta$-linear it preserves independence, and $E$ and $F$ must have the same Hamel dimension. This fact coupled with 5.1 yields some important corollaries.

5.2. Corollary. Let $E$ be infinite dimensional. If $E$ is bornological and quasicomplete $\theta$ must be continuous.

Proof. If $\theta$ is not continuous then the bornological topology on $E$ must be the same as the weak topology and $E$ must be quasicomplete in the weak topology. Suppose $E$ has an infinite dimensional bounded subset. Then the closed convex circled hull of this subset is also bounded and complete. It is thus weakly compact. But this would imply that on $F$ the Mackey topology is stronger than the weak topology, which contradicts 5.1. Thus the bounded subsets of $E$ are finite dimensional. Since $E$ is bornological, it must have the core topology (the strongest possible locally convex topology; see $[9$, p. 56]). In this case, however, $F$ would have to be the algebraic dual of $E$ and therefore of higher dimension, which is impossible. The result follows.

\subsection{Corollary. If the least cardinal of a neighborhood base at zero for} $E$, i.e., the character of $E$, is less than the dimension of $E$, then $\theta$ must be continuous.

Proof. Suppose $\theta$ is not continuous. Let $\aleph$ be the character of $E$. Then $E$ has the weak topology and by standard techniques $[9$, p. 53] $E$ may be embedded as a subspace of $X C_{\alpha}(\alpha \in \aleph)$ where each $C_{\alpha}$ is the complex field. Now since $E$ is a subspace of $X C_{\alpha}(\alpha \in \aleph)$ whose dual is $\bigoplus C_{\alpha}(\alpha \in \aleph)$ and of dimension $\aleph$, the dimension of $F$ cannot exceed $\aleph$. But $E$ and $F$ have the same dimension, so the dimension of $E$ cannot exceed $\aleph$. The result follows.

5.4. Corollary. Let $E$ be infinite dimensional and let $E^{-}$be the completion of $E$. Then if $E$ has the same dimension as $E^{-}, \theta$ must be continuous.

Proof. Suppose $\theta$ is not continuous. Then $E$ has the weak topology and its completion must be the algebraic dual of $F$. For infinite dimensional spaces the algebraic dual is always of higher dimension than the space, and since $E$ and $F$ have the same dimension, the result follows.

In the above considerations, we have used the fact that those spaces whose Mackey and weak topologies are identical are also characterized as those spaces 
in whose dual all weakly compact, convex, circled sets are finite dimensional. Another characterization is also useful. If $N$ is a convex circled neighborhood of zero in $E$ let $H(N)$ be the maximal linear subspace of $E$ contained in $N$. Then the Mackey and weak topologies agree in $E$ if and only if in the Mackey topology for $E, H(N)$ is cofinite dimensional (i.e., $E / H(N)$ is finite dimensional) for each convex circled neighborhood $N$ of zero.

5.5. COROLLARY. If $E$ is an infinite dimensional normed space or a metrizable space other than a subspace of $\times C_{n}(n=1,2, \cdots)$, where each $C_{n}$ is the complex field, $\theta$ must be continuous.

Proof. If $E$ is normed the result is clear. If $E$ is metrizable and its Mackey and weak topologies agree, then the techniques of $[9$, p. 53] yield an embedding of $E$ into $\times C_{n}(n=1,2, \cdots)$.

6. The main theorems. Here we shall employ results of the previous sections to obtain some lattice characterizations of Hilbert space. Our first result is an extension of the theorem of Kakutani and Mackey [6], [7] which gives a lattice characterization of Hilbert space among the infinite dimensional Banach spaces.

6.1. THEOREM. Let $E$ be an infinite dimensional complete Mackey space. If there is an orthocomplementation on $C(E)$, then $E$ may be realized as a Hilbert space in such a way that the orthocomplementation on $C(E)$ is the usual one.

Proof. Let $(E,\langle\rangle$,$) be the corresponding \theta$-bilinear space whose existence and basic properties were established in $\S 3$. Then by Corollary $5.4, \theta$ must be continuous. Thus $\langle$,$\rangle is an inner product making E$ a pre-Hilbert space. We shall call the given topology on $E$ the $\tau$ topology and the topology induced on $E$ by the norm derived from $\langle$,$\rangle , the norm topology. Since we already know that \langle$, induces the orthocomplementation on $C(E)=L(E,\langle\rangle$,$) , it will be sufficient to$ show that $E$ is complete in the norm topology, for then $E$ in the norm topology is a Hilbert space and by the Riesz representation theorem [2] has the same dual, $F$, as does $E$ in the $\tau$ topology. But two Mackey topologies must be the same if they yield the same dual.

Now the dual space $F$ also has an inner product $($,$) defined on it and a$ corresponding norm topology, and we shall need some facts concerning the relation between the norms on $E$ and $F$. First it is clear from the definition of $($,$) that$ $\beta: E \rightarrow F$ is an isometry. Let $x \in E$. Then

$$
\|x\|=\sup \{|\langle x, y\rangle|\|\| y \| \leqslant 1\}=\sup \left\{\mid \beta_{y}(x)\|\| \beta_{y} \| \leqslant 1\right\} \text {. }
$$

Thus if $S_{E}$ and $S_{F}$ denote the closed unit balls in $E$ and $F$, respectively, $S_{F}^{\circ}=S_{E}$. In a similar manner, the polar of $S_{E}$ is $S_{F}$. 
Let $C$ be a weakly compact convex circled subset of $F$. Then $C^{\circ}$ is a neighborhood of zero in $E$ in the $\tau$ topology. By the preceding paragraph, the dual of $E$ in the norm topology includes $F$ and since both norm and $\tau$ topologies are Mackey, the norm topology must be stronger than the $\tau$ topology. Thus we may choose a positive number $\lambda$ small enough so $\lambda S_{E} \subset C^{\circ}$. Then

$$
C=C^{\circ} \subset\left(\lambda S_{E}\right)^{\circ}=\lambda^{-1} S_{E}^{\circ}=\lambda^{-1} S_{F} .
$$

It follows that $C$ is norm bounded in $F$. We conclude that all the weakly compact convex circled subsets of $F$ are norm bounded.

Now let $\left\{x_{n}\right\}_{n=1}^{\infty}$ be a sequence from $E$ which is Cauchy in the norm topology. Let us consider the elements of $E$ as functionals on $F$. Then for each $u \in F$ let $\lim _{n \rightarrow \infty} x_{n}(u)=x(u)$. This defines a linear functional $x$ on $F$. Using the Cauchy-Schwarz inequality and the fact that if $u=\beta_{y}$ then $x_{n}(u)=\beta_{y}\left(x_{n}\right)=$ $\left\langle x_{n}, y\right\rangle$, it is not difficult to see that $\left\{x_{n}\right\}_{n=1}^{\infty}$ as a sequence of functionals on $F$ is uniformly Cauchy on norm bounded subsets of $F$ and hence must converge uniformly to $x$ on such norm bounded sets. Since each $x_{n}$ is weakly continuous on $F$ and since each weakly compact convex circled subset of $F$ is norm bounded, it follows that $x$ is weakly continuous on each weakly compact convex circled subset of $F$. It is then a consequence of the Grothendieck dual characterization of completeness $\left[9\right.$, p. 149, Corollary 2] that $x \in E$. It remains to show that $\left\{x_{n}\right\}_{n=1}^{\infty}$ converges to $x$ in the norm topology. It has already been observed that if $B$ is any norm bounded subset of $F$ then $\left\{x-x_{n}\right\}_{n=1}^{\infty}$ converges uniformly to zero on $B$. If we let $B=\left\{\beta_{\left(x-x_{n}\right)}\right\}_{n=1}^{\infty}$ the desired result follows. Thus $E$ is a Hilbert space under $\langle$,$\rangle , and the theorem is proved.$

We have stated this theorem for Mackey spaces only, but it has consequences of importance for any infinite dimensional complete locally convex space. If $E$ is infinite dimensional and complete, then it is still complete in its Mackey topology (see $[9$, p. 149, Corollary 2]) and $C(E)$ is unchanged so the theorem applies indirectly and still indicates that if an orthocomplementation is possible on $C(E)$ then $C(E)$ comes from a Hilbert space. This observation suggests how the theorem may be applied to a space that is not complete, provided it is complete in its Mackey topology.

One must naturally ask whether 6.1 could be extended to apply to incom ${ }^{-}$ plete Mackey spaces. On this question, Kakutani and Mackey [6] have given examples of incomplete normed spaces which allow orthocomplementations on their lattices of closed subspaces. Thus, 6.1 appears to have reached a natural limit. With stronger assumptions we can, however, obtain results applicable to incomplete Mackey spaces. 
6.2.THEOREM. Let $E$ be a Mackey space and suppose that either on $E$ or on $F$ the Mackey topology is stronger than the weak topology. If there is an orthocomplementation on $C(E)$ under which $C(E)$ is a logic, then $E$ may be realized as a Hilbert space in such a way that the orthocomplementation is the usual one.

Proof. By $5.1, \theta$ is continuous and $E$ is a pre-Hilbert space under $\langle$,$\rangle . By$ $4.1,(E,\langle\rangle$,$) is Hilbertian. It is now a consequence of a lemma due to Piron and$ Araki $[11,7.42]$ that $E$ is complete and a Hilbert space under $\langle$,$\rangle . The remainder$ of the argument is as in the first paragraphs of the proof of 6.1 .

By employing the corollaries of 5.1, further results like 6.2 are possible. We shall leave this to the reader except for the case of metrizable spaces. We begin with the following lemma on normalizability.

6.3. LemmA. Let $V$ be a vector space over $K$. Then if $(V,\langle\rangle$,$) is a \theta$-bilinear space and $J$ is the fixed field under $\theta$, every element of $J$ is of the form $\pm a \theta(a)$, $a \in K$. As a consequence, for every nonzero element $x \in V$ there is $a \lambda \in K$ with $\langle\lambda x, \lambda x\rangle=1$ or -1 .

Proof. Let $b \in J$ and let $a$ be a square root of $b$ in $K$. Then since the coefficients of $x^{2}-b=0$ are fixed by $\theta, \theta$ must permute $\{a,-a\}$. If $\theta(a)=a$ then $b=a \theta(a)$, while if $\theta(a)=-a$ then $b=-a \theta(a)$. If there is no square root for $b$, then let $a$ be a square root of $-b$ and apply the same argument to obtain $b= \pm a \theta(a)$. Thus $J$ consists of all elements $\pm a \theta(a), a \in K$. Now let $x \in V$ with $x \neq 0$. Then $\langle x, x\rangle \in J$. Thus for some $a \in K,\langle x, x\rangle=a \theta(a)$ or $\langle x, x\rangle=-a \theta(a)$. With $\lambda=a^{-1}$, we have $\langle\lambda x, \lambda x\rangle=1$ or -1 .

Now let $E$ be an infinite dimensional metrizable locally convex space over the complex field $C$ and suppose there is an orthocomplementation on $C(E)$ with a corresponding automorphism $\theta$ which is discontinuous. Then by $5.3, E$ is of countable dimension. Let $\left\{x_{n}\right\}_{n=1}^{\infty}$ be a basis for $E$. By copying the GramSchmidt process, we may assume that the set $\left\{x_{n}\right\}_{n=1}^{\infty}$ is orthogonal. Further by 6.3, we may assume that $\left\langle x_{n}, x_{n}\right\rangle= \pm 1$ for all $n$. But if $\left\langle x_{n}, x_{n}\right\rangle=1$ and $\left\langle x_{m}, x_{m}\right\rangle$ $=-1$, then $\left\langle x_{n}-x_{m}, x_{n}-x_{m}\right\rangle=0$ which is inconsistent with the definiteness of $\langle$,$\rangle . Thus by multiplying \langle$,$\rangle by -1$ if necessary, we may assume that $\left\{x_{n}\right\}_{n=1}^{\infty}$ is an orthonormal set. An element of $E$ in this basis is, of course, a sum $\Sigma \lambda_{n} x_{n}$ where all but finitely many of the $\lambda$ 's are zero. Further, and more importantly, if $\Sigma \mu_{n} x_{n}$ is another such element

$$
\left\langle\sum \lambda_{n} x_{n}, \sum \mu_{n} x_{n}\right\rangle=\sum \lambda_{n} \theta\left(\mu_{n}\right) .
$$

Let $A$ be the space of finitely nonzero sequences $\left\{\lambda_{n}\right\}_{n=1}^{\infty}$ of complex numbers and define $\langle$,$\rangle on A$ by 


$$
\left\langle\left\{\lambda_{n}\right\}_{n=1}^{\infty},\left\{\mu_{n}\right\}_{n=1}^{\infty}\right\rangle=\sum \lambda_{n} \theta\left(\mu_{n}\right),
$$

and let $A$ have the topology induced by $\langle$,$\rangle . Then it is clear that \Sigma \lambda_{n} x_{n} \rightarrow$ $\left\{\lambda_{n}\right\}_{n=1}^{\infty}$ is an isomorphism of $E$ onto $A$ which preserves $<$, 's and that since the topologies on $E$ and $A$ are determined by the $\langle$,$\rangle 's this isomorphism is topological.$ A little reflection shows that in fact the functionals $\left\langle\circ,\left\{\mu_{n}\right\}_{n=1}^{\infty}\right\rangle$ on $A$ form a set which is independent of the choice of $\theta$. Thus the topology on $A$ is independent of $\theta$, and $A$ is, up to isomorphism, the unique infinite dimensional metrizable locally convex space which allows orthocomplementations with discontinuous $\theta$ on its lattice of closed subspaces. Further any orthocomplementation on $C(A)$ can be realized up to isomorphism in a canonical way by $(\dagger)$.

Let $($, ) be the $\theta$-bilinear form resulting from $(\dagger)$ when $\theta$ is conjugation on $C$. Then under ( , ), $A$ is a pre-Hilbert space. Let $H$ denote the completion of $A$ to a Hilbert space. At this point it is convenient to change the notation for sequences. If $\left\{\lambda_{n}\right\}_{n=1}^{\infty}$ is a sequence of complex numbers we shall denote it by $\left(\lambda_{n}\right)$. Then $H$ consists of all sequences $\left(\lambda_{n}\right)$ with $\Sigma \lambda_{n} \bar{\lambda}_{n}<\infty$ and $A$ consists of the finitely nonzero $\left(\lambda_{n}\right)$. Let us call an element $x$ of $H$ or $A$ rational if in $x=$ $\left(\lambda_{n}\right)$ each $\lambda_{n}$ is a rational number. We shall need some facts concerning rational elements of $H$ and $A$.

6.4. LEMmA. Let $x$ and $\left\{z_{i}\right\}_{i=1}^{n}$ be rational elements of $H$ such that $x \perp z_{i}$ for $i=1,2, \cdots, n$ and such that $\left(z_{i}, z_{j}\right)$ is rational for $i, j=1,2, \cdots, n$. Then for any $\epsilon>0$ there is a rational element $y$ of $A$ such that $\|x-y\|<\epsilon$ and $y \perp z_{i}$ for $i=1,2, \cdots, n$.

Proof. The proof is by induction on $n$. In case $n=1$, let $\left\{u_{m}\right\}_{m=1}^{\infty}$ and $\left\{v_{m}\right\}_{m=1}^{\infty}$ be rational sequences from $A$ converging to $x$ and $z_{1}$, respectively. Then if $y_{m}=u_{m}-\left(u_{m}, z_{1}\right)\left(v_{m}, z_{1}\right)^{-1} v_{m},\left\{y_{m}\right\}_{m=1}^{\infty}$ is also a rational sequence from $A$ which converges to $x$. Further $y_{m} \perp z_{1}$ for each $m$. Thus we may take $y=y_{m}$ for a suitably large $m$ to obtain the desired result.

Let us assume the assertion is true for the case $n-1$ and let $x$ and $\left\{z_{i}\right\}_{i=1}^{n}$ satisfy the stated hypotheses. If the $\left\{z_{i}\right\}_{i=1}^{n}$ were a linearly dependent set, the problem would reduce to the case $n-1$, so we may assume that $\left\{z_{i}\right\}_{i=1}^{n}$ is a linearly independent set. For any $r \in H$ and any positive integer $k$ let $(r)^{k}$ be that element of $H$ which is identical to $r$ on the first $k$ coordinates but after the $k$ th position is zero on each coordinate. Let $(r)_{k}=r-(r)^{k}$. Then regardless of the choice of $r$ it is clear that as $k$ goes to infinity $\left\|(r)_{k}\right\|=\left\|r-(r)^{k}\right\| \rightarrow 0$. Let $\epsilon>0$. Choose $k_{0}$ large enough so that $\left\|(x)_{k_{0}}\right\|<\epsilon / 6 n$. Now define recursively $p_{1}=z_{1}$ and

$$
p_{i}=z_{i}-\sum_{j=1}^{i-1}\left(z_{i}, p_{j}\right)\left(p_{j}, p_{j}\right)^{-1} p_{j}
$$


for $i=1,2, \cdots, n$. Then the $p_{i}$ 's are rational and mutually orthogonal and $\left\|p_{i}\right\|^{2}$ is rational for each $i$. Since the set $\left\{z_{i}\right\}_{i=1}^{n}$ is independent, no $p_{i}$ is zero. Let $\mu=(1 / 3) \min _{1<i<n}\left\|p_{i}\right\|$. Now using the induction assumption (validity at $n-1)$, choose for each $i, 1 \leqslant i \leqslant n$, a rational $q_{i}$ from $A$ such that $\left\|p_{i}-q_{i}\right\|<\mu$ and $q_{i} \perp p_{j}$ for all $j \neq i, 1 \leqslant j \leqslant n$. By expanding $\left\|p_{i}-q_{i}\right\|^{2}<\mu^{2}$ and substituting from $\left\|q_{i}\right\|-\mu<\left\|p_{i}\right\|$ one may obtain $\left\|q_{i}\right\|\left(\left\|q_{i}\right\|-\mu\right)<\left(q_{i}, p_{i}\right)$. Using $\left\|p_{i}\right\|-$ $2 \mu<\left\|q_{i}\right\|-\mu$ leads to $\left\|q_{i}\right\|\left(\left\|p_{i}\right\|-2 \mu\right)<\left(q_{i}, p_{i}\right)$. Let us define

$$
\lambda_{i}=\left((x)_{k_{0}}, p_{i}\right) /\left(q_{i}, p_{i}\right) \text { for } i=1,2, \cdots, n \text {. }
$$

Then

$$
\left\|\lambda_{i} q_{i}\right\|\left(\left\|p_{i}\right\|-2 \mu\right)<\left|\left(\lambda_{i} q_{i}, p_{i}\right)\right|=\left|\left((x)_{k_{0}}, p_{i}\right)\right| \leqslant(\epsilon / 6 n)\left\|p_{i}\right\|
$$

by the Cauchy Schwarz inequality. It follows that

$$
\left\|\lambda_{i} q_{i}\right\|<(\epsilon / 6 n)\left[\left\|p_{i}\right\| /\left(\left\|p_{i}\right\|-2 \mu\right)\right] \leqslant \epsilon / 2 n
$$

for $i=1,2, \cdots, n$. Let

$$
y=(x)^{k} 0+\sum_{i=1}^{n} \lambda_{i} q_{i}
$$

Then $y$ is a rational element of $A$ and

$$
\|x-y\|=\left\|(x)_{k_{0}}-\sum_{i=1}^{n} \lambda_{i} q_{i}\right\| \leqslant\left\|(x)_{k_{0}}\right\|+\sum_{i=1}^{n}\left\|\lambda_{i} q_{i}\right\| \leqslant(\epsilon / 6 n)+n(\epsilon / 2 n)<\epsilon .
$$

Further

$$
\begin{aligned}
\left(y, p_{i}\right) & =\left((x)^{k_{0}}, p_{i}\right)+\sum_{j=1}^{n} \lambda_{j}\left(q_{j}, p_{i}\right)=\left((x)^{k_{0}}, p_{i}\right)+\lambda_{i}\left(q_{i}, p_{i}\right) \\
& =\left((x)^{k_{0}}, p_{i}\right)+\left((x)_{k_{0}}, p_{i}\right)=\left(x, p_{i}\right)=0
\end{aligned}
$$

for each $i$. Since each $z_{i}$ is a linear combination of the $p_{i}$ 's it follows that $y \perp z_{i}$ for each $i$ and this completes the proof.

6.5. Lemma. Let $x_{0}$ and $z_{0}$ be rational orthogonal elements of $H$ with $\left\|x_{0}\right\|^{2}$ and $\left\|z_{0}\right\|^{2}$ both rational. Then there are sequences $\left\{x_{n}\right\}_{n=1}^{\infty}$ and $\left\{z_{n}\right\}_{n=1}^{\infty}$ of rational elements of $A$ which satisfy

(i) $x_{n} \perp z_{m}(n, m=0,1,2, \cdots)$;

(ii) $x_{n} \rightarrow x_{0}$ and $z_{n} \rightarrow z_{0}$.

This lemma is closely related to Lemma 7.41 of [11]. The proof is easily constructed using 6.4.

We are now prepared to deal with logics on metrizable spaces. 
6.6. THEOREM. Let $E$ be an infinite dimensional metrizable locally convex space. If there is an orthocomplementation on $C(E)$ under which $C(E)$ is a logic, then $E$ may be realized as a Hilbert space in such a way that the orthocomplementation is the usual one.

Proof. This result will follow in the same way as 6.2 , provided the automorphism $\theta$ is shown to be continuous. The only possible exception to this is the space $A$ and it will be sufficient to show that $\mathrm{C}(A)$ is not converted to a logic by any $\theta$-bilinear symmetric form $\langle$,$\rangle defined as in ( \dagger$ ).

Let us again consider the space $A$ with the inner product $($,$) and its Hilbert$ space completion $H$. Let $x_{0}=\left(\lambda_{n}\right)$ be a rational element of $H$ with $\left\|x_{0}\right\|=1$ and $\lambda_{n} \neq 0$ for each $n$. Let $y=\left(\mu_{n}\right)$ where $\mu_{1}=\lambda_{1}^{-1}$ and $\mu_{n}=0$ for $n>1$ and let $z_{0}=y-x_{0}$. Then $x_{0}$ and $z_{0}$ are rational orthogonal elements of $H$ with both $\left\|x_{0}\right\|^{2}$ and $\left\|z_{0}\right\|^{2}$ rational. Let $\left\{x_{n}\right\}_{n=1}^{\infty}$ and $\left\{z_{n}\right\}_{n=1}^{\infty}$ be the sequences whose existence is assured by 6.5. Then with $M$ equal to the lattice sum of the spaces $C \circ x_{n}(n=1$, $2, \cdots)$, the proof of $[11,7.42]$ shows that $A \neq M+M^{\perp}$. Thus $(A,()$,$) is not$ Hilbertian. Now we claim that, by their definitions, $M$ and $M^{\perp}$ are completely independent of which $\theta$-bilinear space $(A,\langle\rangle$,$) is used provided \langle$,$\rangle has the canon-$ ical form ( $\dagger$ ). First $M$ is the closure of the linear hull of the set $\left\{x_{n}\right\}_{n=1}^{\infty}$ in the topology induced on $A$ by any $(A,\langle\rangle$,$) , since as pointed out above they all induce$ the same topology on $A$. Next

$$
M^{\perp}=\left(\bigvee_{n=1}^{\infty} C \circ x_{n}\right)^{\perp}=\bigwedge_{n=1}^{\infty}\left(C \circ x_{n}\right)^{\perp}
$$

Let $x_{n}=\left(\lambda_{i}\right)$ and $y=\left(\mu_{i}\right) \in A$. Then $x_{n} \perp y$ if and only if $\Sigma \lambda_{i} \theta\left(\mu_{i}\right)=0$, which is true if and only if $\theta\left(\Sigma \lambda_{i} \theta\left(\mu_{i}\right)\right)=\Sigma \lambda_{i} \mu_{i}=0$, because $\theta$ is involutive and the $\lambda_{i}$ are rational. Thus each $\left(C \circ x_{n}\right)^{\perp}$ is independent of $(A,\langle\rangle$,$) and since$

$$
\bigwedge_{n=1}^{\infty}\left(C \circ x_{n}\right)^{\perp}=\bigcap_{n=1}^{\infty}\left(C \circ x_{n}\right)^{\perp}
$$

$M^{\perp}$ is independent of $(A,\langle\rangle$,$) . Since A \neq M+M^{\perp}$ it follows that no $(A,\langle\rangle$, with $\langle$,$\rangle defined in accordance with (\dagger)$ is Hilbertian. Since the lattices $L(A,\langle\rangle$,$) ,$ with $\langle$,$\rangle as in (\dagger)$, with their natural orthocomplementations, include all orthocomplementations on $C(A)$ except for isomorphic copies, there is by 4.1 no orthocomplementation on $\mathrm{C}(A)$ under which $\mathrm{C}(A)$ is a logic. This concludes the proof.

7. An example. The results of the previous section have shown that beyond the Hilbert spaces there are no spaces with well-behaved topologies which also allow the structure of a logic on their lattice of closed subspaces. One may ask if 
in fact there are any spaces other than Hilbert spaces on which logics may be constructed. We shall show here that there are such spaces. From this fact there seems little hope of improving our results.

The construction rests rather heavily on the axiom of choice. By 4.1 and $[11,7.42]$ it is evident that if ${ }^{\prime}$ is an orthocomplementation on $C(E)$ under which $C(E)$ is a logic and if $E$ is not a Hilbert space, then the corresponding field automorphism $\theta$ must be discontinuous. Thus we are restricted to the complex field. Clearly the first step is to find a suitable discontinuous automorphism $\theta$ on $E$. This can be done in many ways but significantly not without the use of the axiom of choice [11, p. 45], [10]. Let $\varphi$ stand for the usual conjugation automorphism on $C$ and let $\alpha$ be any discontinuous automorphism on $C$. Then we may let $\theta=$ $\alpha \varphi \alpha^{-1}$. Now let $H$ be any complex Hilbert space with its standard inner product ( , ). Let $H_{\alpha}$ be the same set as $H$ with the same addition but with a new scalar multiplication $*$ defined by $a * x=\alpha^{-1}(a) x$ for all $a \in C$ and $x \in H_{\alpha}(=H)$. Define $\langle$,$\rangle on H_{\alpha}$ by $\langle x, y\rangle=\alpha((x, y))$. Then, for any $a, b \in C$ and $x, y \in H_{\alpha}$,

$$
\begin{aligned}
\langle a * x, b * y\rangle & =\alpha\left(\left(\alpha^{-1}(a) x, \alpha^{-1}(b) y\right)\right)=\alpha\left(\alpha^{-1}(a)(x, y) \varphi \alpha^{-1}(b)\right) \\
& =a \alpha((x, y)) \alpha \varphi \alpha^{-1}(b)=a\langle x, y\rangle \theta(b)
\end{aligned}
$$

and

$$
\langle x, y\rangle=\alpha((x, y))=\alpha \varphi((y, x))=\left(\alpha \varphi \alpha^{-1}\right) \alpha((y, x))=\theta(\langle y, x\rangle) .
$$

Thus $\left(H_{\alpha},\langle\rangle,\right)$ is a $\theta$-bilinear space. Since $x \perp y$ in $H$ if and only if $x \perp y$ in $H_{\alpha}$, it is clear that the identity map from $H$ to $H_{\alpha}$ induces an isomorphism between $L(H,()$,$) and L\left(H_{\alpha},\langle\rangle,\right)$. Thus the lattice $L\left(H_{\alpha},\langle\rangle,\right)$ with its orthocomplementation is isomorphic to the logic of a Hilbert space. The space $H_{\alpha}$ with its Mackey topology is however not a Hilbert space by the results of $\S \S 5$ and 6 .

Using the technique just outlined one may produce a large class of non-Hilbert spaces which provide for the construction of all the logics that may be obtained from the Hilbert spaces. Whether there exist non-Hilbert spaces with logics which are not isomorphic to the logic of a Hilbert space is, to our knowledge, an open question. This question appears to be related to a question of Varadarajan [11, p. 176], namely: Can every logic be embedded in a standard logic? An answer to either question would provide an illuminating commentary on Piron's theorem [11, 7.44].

8. Question models. The foregoing lattice characterizations of Hilbert space have some significance for the foundations of quantum theory. In particular, within the context of the locally convex spaces they allow us to demonstrate the 
unique suitability of the Hilbert spaces as a source of question models. Let us make this concept of suitability precise. If the lattice of closed subspaces of a locally convex space should correspond to the physically meaningful questions then the one dimensional questions would have a meaning as well as the pure states of the system. The most searching question one cais ask is of the pure state of the system; hence, such questions on the one hand may be identified with the pure states while on the other hand being atomic must be one dimensional subspaces of the vector space. With this identification the topology on the vector space yields a natural quotient topology for the pure states. There is also a natural metric topology for the states. If $\varphi$ and $\psi$ are states and $L$ is the lattice of questions, $d(\varphi, \psi)$ is defined by

$$
d(\varphi, \psi)=\sup |\varphi(q)-\psi(q)| \quad(q \in L) .
$$

We shall say that a locally convex space $E$ is suitable for quantum theory if:

$S_{1}$. There is an isomorphism $\Gamma$ from $C(E)$ to the lattice $L$ of physically meaningful questions.

$S_{2}$. The metric topology for the pure states agrees with the quotient topology for the pure states obtained from $E$ through the identification $\Gamma$.

Using the theorem of Gleason [4] on states, it is not difficult to show that if $E$ is a separable Hilbert space and $\Gamma$ is the correspondence generally assumed in nonrelativistic quantum mechanics, then $\mathrm{S}_{2}$ is satisfied and $E$ is suitable for quantum theory by our criteria. By the same means, it may be shown for any Hilbert space that if $\Gamma$ exists then $S_{2}$ is also satisfied. In order to conclude that the Hilbert spaces are the only locally convex spaces that can possibly satisfy $S_{1}$ and $S_{2}$ we must of course make assumptions about reality; but when chosen to take maximum advantage of our results only one is necessary and we believe it has some natural validity.

The necessary assumption is the following:

$\mathrm{N}$. The lattice $L$ of questions in quantum theory is a logic.

This is generally deemed a technical necessity [3], [5], [8], [11]. In addition when combined with $S_{1}$ and the results of $\S 6$ it has the effect of eliminating from further consideration most spaces of interest. Beyond the Hilbert spaces the only spaces that might allow a logic structure are a certain class of spaces which admit only the weak topology, are incomplete, and are nonmetrizable. Some examples are constructed in $\$ 7$. These spaces are yet to be considered in relation to $S_{2}$.

The nonmetrizability of the non-Hilbert spaces which allow logics provides the necessary contact with $S_{2}$. Our argument will be completed by showing that 
if $S$ represents the collection of one dimensional subspaces of $E$ with the quotient topology and if $S$ is metrizable then $E$ must also be metrizable. For any nonzero $x \in E$ let $l(x)$ denote the subspace determined by $x$ and if $U$ is a convex circled neighborhood of zero in $E$ let

$$
M(x, U)=\bigcup\{\lambda(x+U) \mid \lambda \in K\}
$$

and let $L(x, U)$ denote the collection of elements of $S$ which are contained in $M(x, U)$. Clearly the family of $L(x, U)$ 's is a neighborhood base for $l(x)$ in $S$. Now let $x_{0}$ and $y_{0}$ denote independent elements of $E$. Assume $S$ is metrizable and let $\left\{U_{n}\right\}_{n=1}^{\infty}$ and $\left\{U_{n}\right\}_{n=1}^{\infty}$ be bases at $l\left(x_{0}\right)$ and $l\left(y_{0}\right)$ in $S$. For each $(n, m)$ let $U(n, m)$ be a convex, circled, open neighborhood of zero in $E$ with $L\left(x_{0}, U(n, m)\right) \subset U_{n}$ and $L\left(y_{0}, U(n, m)\right) \subset V_{m}$. Now let $U$ be any convex, circled, open neighborhood of zero in $E$. Choose a positive integer $n$ large enough so that $(1 / n) x_{0} \in(1 / 3) U$. Again choose a convex, circled, open neighborhood $V$ of zero in $E$ such that $\sup \left\{|\lambda| \mid \lambda x_{0}+\mu y_{0} \in V\right\} \leqslant(1 / n+1)$ and $\sup \left\{|\mu| \mid \lambda x_{0}+\right.$ $\left.\mu y_{0} \in V\right\} \leqslant(1 / n+1)$. Let $W=[(1 / 2) V] \cap[(1 / 3) U]$. Then a lengthy but elementary calculation shows that

$$
M\left(x_{0}, W\right) \cap\left(M\left(y_{0}, W\right)+\left(x_{0}-y_{0}\right)\right) \subseteq U+x_{0} .
$$

Let $U_{p} \subseteq M\left(x_{0}, W\right)$ and $V_{q} \subseteq M\left(y_{0}, W\right)$. Then

$$
M\left(x_{0}, U(p, q)\right) \cap\left(M\left(y_{0}, U(p, q)\right)+\left(x_{0}-y_{0}\right)\right) \subseteq U+x_{0} .
$$

Since for each $p$ and $q$ the set $M\left(x_{0}, U(p, q)\right) \cap\left(M\left(y_{0}, U(p, q)\right)+\left(x_{0}-y_{0}\right)\right)$ is a convex open neighborhood of $x_{0}$ it follows that $E$ has a countable base at $x_{0}$. Thus $E$ is metrizable. It now follows from 6.6 that the only spaces that can possibly satisfy both $S_{1}$ and $S_{2}$ are the Hilbert spaces. We conclude that the Hilbert spaces are the only locally convex spaces which are suitable for the construction of question models in quantum mechanics.

\section{BIBLIOGRAPHY}

1. B. H. Arnold, Rings of operators on vector spaces, Ann. of Math. (2) 45 (1944), 24-49. MR 5, 147.

2. G. Bachman and L. Narici, Functional analysis, Academic Press, New York, 1966. MR 36 \#638.

3. G. Birkhoff and J. von Neumann, The logic of quantum mechnics, Ann. of Math. 37 (1936), 823-843.

4. A. M. Gleason, Measures on the closed subspaces of a Hilbert space, J. Math. Mech. 6 (1957), 885-893. MR 20 \#2609.

5. J. Jauch, Foundations of quantum mechanics, Addison-Wesley, Reading, Mass., 1968. MR 36 \#1151. 
6. S. Kakutani and G. W. Mackey, Two characterizations of real Hilbert space, Ann. of Math. (2) 45 (1944), 50-58. MR 5, 146.

7. Ring and lattice characterizations of complex Hilbert space, Bull. Amer. Math. Soc. 52 (1946), 727-733. MR 8, 31.

8. G. W. Mackey, The mathematical foundations of quantum mechanics: A lecturenote volume, Benjamin, New York, 1963. MR 27 \#5501.

9. H. Schaefer, Topological vector spaces, Macmillan, New York, 1966. MR 33 \#1689.

10. R. Solovay, The measure problem, Notices Amer. Math. Soc. 12 (1965), 217, Abstract \#65T-62.

11. V. S. Varadarajan, Geometry of quantum theory. Vol. 1, Van Nostrand, Princeton, N. J., 1968.

DEPARTMENT OF MATHEMATICS, ANDREWS UNIVERSITY, BERRIEN SPRINGS, MICHIGAN 49104

Current address: 11209 Rosarita Drive, Loma Linda, California 92354 